\title{
Hau(p)tsache Kinder: Die phytotherapeutische Apotheke für die Kinderhaut
}

\author{
Karoline Fotinos-Graf \\ Praxis Aromaphyt, Bern, Schweiz
}

\section{Einleitung}

Das Gebiet der dermatologischen Erkrankungen bei Kindern nimmt in der Beratung in der Apotheke einen wichtigen Platz ein. Im Folgenden sollen für diverse pädiatrisch-dermatologische Erkrankungen einige ausgewählte Arzneipflanzen vorgestellt werden.

\section{Atopische Dermatitis, Ekzeme}

Oenothera biennis (Nachtkerze) ist eine der am besten untersuchten Arzneipflanzen bei atopischer Dermatitis [1] Therapeutisch genutzt wird das Öl der Samen, dessen hauptsächliche Inhaltsstoffe die essenziellen Fettsäuren Linolsäure (60-80\%) und $\gamma$-Linolensäure (8-14\%) sind [2, S. 232].

Atopiker weisen häufig einen Mangel an $\gamma$-Linolensäure auf, was eine verminderte Herstellung immunregulierender/ entzündungshemmender Prostaglandine zur Folge hat [2, S. 233; 3]. Durch systemische Zuführung von $\gamma$-Linolensäure kann dieser Mangel reduziert werden [4, S. 315]. Wichtig ist eine ausreichend hohe Dosierung (1-12 Jahre: 2-mal täglich 2 Kapseln à 1000 mg Nachtkerzenöl; ab 12 Jahren: 2-mal 3 Kapseln) sowie eine Mindestdauer von 8-12 Wochen. Nachtkerzenöl kann auch im Rahmen der topischen Basistherapie appliziert werden.

Die gewünschte antiinflammatorische Wirkung von Cardiospermum halicacabum (Herzsamen) kommt durch Phytosterole zustande, die in die Arachidonsäurekaskade eingreifen und so die Entstehung von Entzündungsmediatoren reduzieren [5, S. 97; 6]. Ausser der antiinflammatorischen Wirkung weist Cardiospermum antipruriginöse und feuchtigkeitsspendende Wirkungen auf [2, S. 69].

Eingesetzt werden Zubereitungen aus 10\%iger homöopathischer Urtinktur [2, S. 70]. Salben/Lotionen können bei leichter atopischer Dermatitis oder juckenden Ekzemen mehrmals täglich aufgetragen werden.

Die antiinflammatorische Wirkung von Hamamelis virginiana (Zaubernuss) bei atopischer Dermatitis wurde durch mehrere Studien belegt [5, S. 260]; ausserdem wirkt die Gerbstoffdroge adstringierend, juckreizstillend und wund- heilungsfördernd [5, S. 259; 7]. Gerbstoffe sind allerdings nur in ethanolisch-wässrigen Auszügen enthalten; das destillierte Hamameliswasser enthält keine Gerbstoffe, ist aber ebenfalls entzündungshemmend [8, S. 250].

Hamamelis wird beispielsweise als Aufguss für Umschläge und Waschungen benutzt (z.B. starke Juckreizattacken, nässende Läsionen) oder als Hamameliswasser formuliert in Cremes oder Salben mehrmals täglich aufgetragen.

\section{Kopfläuse}

Diverse fette Öle, welche die Läuse mechanisch abtöten sollen, kommen infrage (z.B. Kokos-, Raps-, Sojaöl) [4, S. 302], eventuell durch zusätzliche Zugabe geeigneter ätherischer Öle wie z.B. Lavendel (Lavandula angustifolia) oder Eukalyptus (Eucalyptus radiata) [4, S. 303; 9, S. 506].

Genutzt wird auch das Öl aus Samen und Blättern von Azadirachta indica (Neembaum), welches unter anderem Azadirachtin enthält. Azadirachtin hemmt die Chitinsynthese, sodass die Metamorphose der Laus nicht komplett stattfinden kann. Neemöl hat zudem eine gewisse RepellentWirkung [4, S. 303].

\section{Varizella (Windpocken)}

Empfohlen werden Waschungen und Umschläge mit Gerbstoffdrogen, z.B. Hamamelis oder Schwarztee [4, S. 321]. Bei wundgekratzten Bläschen kann eine Hamamelisoder Ringelblumencreme eingesetzt werden [4, S. 321].

Auch diverse ätherische Öle kommen aufgrund ihrer ausgezeichnet antiinfektiös sowie antiphlogistisch und juckreizstillend wirkenden Inhaltsstoffe als Massnahme infrage, z.B. Lavendelöl, Cistrosenöl, Teebaumöl sowie Palmarosaöl [9, S. 495-496; 10, S. 82, 246, 334, 431; 11, S. 122] (Tab. 1).

\section{Windeldermatitis, Windelsoor}

Calendula officinalis (Ringelblume) hat antiphlogistische, wundheilungsfördernde sowie antibakterielle und fungista-

\section{KARGER}

Fax +497614520714

Information@Karger.com

www.karger.com 
Tab. 1. Mögliche Rezeptur (Dosierung: 1- bis 2-mal täglich lokal dünn auf die Pusteln auftragen)

\begin{tabular}{ll}
\hline & $\mathrm{ml}$ \\
\hline Lavendel (Lavandulae angustifoliae aetheroleum) & 1,00 \\
Palmarosa (Cymbopogoni martinii aetheroleum) & 0,75 \\
Cistrose (Cisti ladaniferi aetheroleum) & 0,75 \\
Teebaum (Melaleucae alternifoliae aetheroleum) & 0,50 \\
Ringelblumenöl (Calendulae extractum oleosum) ad & 100,00 \\
\hline
\end{tabular}

tische Eigenschaften [2, S. 267; 12]. In der Akutphase eignen sich Waschungen/Sitzbäder mit Aufgüssen oder verdünnter Tinktur (2-4 ml Tinktur auf 0,25-0,5 1 Wasser) [4, S. 280]. Zur Vorbeugung können Calendulacremes als tägliche Pflege benutzt werden.

Waschungen oder Bäder können vor allem bei nässenden Läsionen auch mit Gerbstoffdrogen erfolgen, z.B. Eichenrinde, Hamamelis oder Schwarztee sowie mit Kamille (Matricaria chamomilla) [13, S. 146].

Zudem eignen sich ebenfalls diverse antimykotische ätherische Öle, wie z.B. das auch für Babyhaut unbedenkliche Lavendelöl [9, S. 206], verdünnt in Ringelblumenöl (0-1 Jahr 0,5\%, 1-3 Jahre 1\%, ab 3 Jahren 2\%).

\section{Verbrennungen, Sonnenbrand, Wunden}

Die antiphlogistische Wirkung von Matricaria recutita (Kamille) ist gut belegt [2, S. 179]; ausserdem hat Kamille wundheilungsfördernde, antibakterielle und antimykotische Eigenschaften [5, S. 138]. Für die Akutphase bei Verbrennungen und Sonnenbrand können kühlende Umschläge mit einem Aufguss aus 1 EL (ca. 2,5 g) Kamillenblüten/150 ml Wasser gemacht werden, eventuell verstärkt mit $5 \mathrm{ml} \mathrm{Kamil-}$ lentinktur [4, S. 290].

Diese Zubereitung eignet sich auch zur Wundreinigung [14, S. 421]; für eine Wunddesinfektion bietet sich verdünn- te Kamillentinktur (1:1-1:2) an [2, S. 1153]. Für nässende Wunden kann eine weisse Schüttelmixtur mit 2\% Kamillenfluidextrakt hergestellt werden [4, p. 278; 14, S. 437].

Lavendelöl (L. angustifolia) ist ein ausgezeichnetes ätherisches Öl bei Verbrennungen und darf als eines der wenigen Öle kleinflächig auch unverdünnt aufgetragen werden [9, S. 206]. Bei Sonnenbrand, da grossflächig, empfiehlt sich jedoch eine Verdünnung (Verdünnung 0-1 Jahr 0,5\%, 1-3 Jahre 1\%, ab 3 Jahren 2\%), z.B. eingearbeitet in ein AloeVera-Gel [9, S. 447].

Lavendelöl sowie andere ätherische Öle eignen sich $\mathrm{zu}$ dem auch für die Wundbehandlung [15], viele davon auch für Kinder [16].

H. virginiana (Zaubernuss) wird als Aufguss oder Hamameliswasser für kühlende Umschläge bei Verbrennungen eingesetzt; für die Wundbehandlung eignet sich Hamamelis aufgrund der Gerbstoffe bei nässenden Wunden oder zur Blutstillung als Aufguss oder als verdünnter Extrakt [4, S. 275].

Für tiefere oder schlecht heilende Wunden eignen sich Zubereitungen aus Ringelblumenblüten [14, S. 421] (Aufguss/verdünnte Tinktur, Creme/Salbe, Ringelblumenöl: je nach Akuität).

\section{Fazit}

Ein breites Spektrum an Arzneipflanzen steht für die Behandlung dermatologischer Erkrankungen bei Kindern zur Verfügung. Wichtig sind aber auch Art der Anwendungen und, gerade in der Dermatologie, deren Galenik, da die richtige Galenik den therapeutischen Erfolg optimieren kann [17].

\section{Disclosure Statement}

Es besteht kein Interessenkonflikt.

\section{Literatur}

1 Bachmann C: Wirksame Arzneipflanzen bei dermatologischen Erkrankungen. Ars Medici Thema Phytotherapie 2011;335-337.

2 Schilcher H, Kammerer S, Wegener T: Leitfaden Phytotherapie. München, Elsevier, 2010.

3 Invernizzi C: Die Rolle von Nachtkerzensamenöl bei chronisch-entzündlichen Prozessen begleitet von einem Gamma-Linolensäure-Mangel. Schweiz Z Ganzheitsmed 2011;23:149-152.

4 Bühring U, Ell-Beiser H, Girsch M: Heilpflanzen in der Kinderheilkunde. Stuttgart, Sonntag, 2008.

5 Augustin M, Hoch Y: Phytotherapie bei Hauterkrankungen. München, Elsevier, 2004.

6 Bachmann C: Cardiospermum bei dermatologischen Problemen. Ars medici Thema Phytotherapie 2010;7-8.

7 Welzel J, Abdul Malek F, Zimmermann A, Wolff HH: Hametum Wund- und Heilsalbe bei arzneimittelinduzierten Hautsymptomen. Ars medici Thema Phytotherapie 2010;2-6.
8 Bäumler S: Heilpflanzenpraxis heute: Arzneipflanzenporträts, Band 1. München, Elsevier, 2012.

9 Wabner D, Beier C: Aromatherapie. München, Elsevier, 2009.

10 Wabner D: Taschenlexikon der Aromatherapie. Bad Kötzting/München, Systemische Medizin AG, 2013.

11 Baudoux D: Les cahiers pratiques d'aromathérapie selon l'école française: pédiatrie. Luxembourg, Edition Inspir S.A., 2001.

12 Really Fine Ideas: Mit Pflanzen Wunden versorgen. Schweiz Z Ganzheitsmed 2011;23:134-135.

13 Huber R: Mind-Maps Phytotherapie. Stuttgart, Hippokrates, 2009.

14 Bäumler S: Heilpflanzenpraxis heute: Rezepturen und Anwendung, Band 2. München, Elsevier, 2013.
15 Fotinos-Graf K: Ätherische Öle in der Wundheilung und Entwicklung von geeigneten Rezepturen. 2014. www.smgp.ch/smgp/homeindex/ faehigkeitsprogf/zertifikatsarbeiten/FotinosGrafKaroline.pdf (letzter Zugriff am 1.10.2015).

16 SMGP: Empfehlungen der Schweizerischen Medizinischen Gesellschaft für Phytotherapie zur Verwendung von ätherischen Ölen bei Kleinkindern im Alter < 30 Monaten auf ärztliche Verschreibung. www.smgp.ch/smgp/homeindex/ arzneimittelf/dokumente/Empfehlungsliste_Terpene.pdf (letzter Zugriff am 1.10.2015).

17 Daniels R: Die richtige Galenik für kranke Haut. Pharmazeutische Zeitung online, 2009. www. pharmazeutische-zeitung.de/index.php?id= 30020 (letzter Zugriff am 1.10.2015). 